\title{
Polarization Model for Molten Carbonate Fuel Cell Cathodes
}

\author{
Shigenori Mitsushima*, Satoshi Kuroe, Shimpei MATSUdA ${ }^{\dagger}$ and Tomoichi KAMO
}

Received February 6, 1998 ; Accepted May 7, 1998

\begin{abstract}
High performance is required during over 40 thousand hours of operation for practical use of the molten carbonate fuel cell in power generation systems. In order to achieve such performance, the cathode pore structure design which defines the cathode polarization must be optimized. A polarization model for the cathode reaction has been investigated to understand the relationship between the pore size distribution, the electrolytefill level, and the polarization. The electrodes with smaller pore diameter have smaller polarization and a wider suitable electrolyte fill level due to their larger reaction surface areas. The reaction current profile in the suitable electroly te fill level is determined by the balance of the ionic resistivity and the reaction surface area.
\end{abstract}

\section{INTRODUCTION}

Molten carbonate fuel cell (MCFC) power generation systems have been developed as an environment-friendly power generation system. High performance is required throughout over 40 thousand hours of operation for practical cell use. The factors limiting lifetime are ascribed to electrolyte loss by hardware corrosion, short circuiting of the nickel deposited in the electrolyte plate, and pore structure change of the cathode and the electrolyte matrix ${ }^{1-4)}$.

Cathode polarization of MCFCs is one of the most important factors in understanding the cell performance ${ }^{1)}$, because it is about half of the total polarization and, it is sensitive to the electrolyte fill level and to the pore size distribution. To develop a high performance MCFC, a cathode which has a low reaction polarization and low

Hitachi Research Laboratory, Hitachi, Ltd. (1-1, Omika-cho 7-chome, Hitachi-shi, lbaraki - ken, 319-1292 Japan)

tCorporate Research \& Development Promotion Office, Hitachi, Ltd. (5-1, Marunouchi 1-chome, Chiyoda-ku, Tokyo-to, 100-0005, Japan)

Key Words: Fuel Cell, Molten Carbonate, Cathode, Polarization Model dependence on the electrolyte fill level is prerequisite.

The MCFC cathode is a porous gas diffusion electrode. Several gas diffusion electrode models have been developed to understand the behavior of the cathode polarization 5il. One is the homogeneous cylinder model ${ }^{5)}$ and another is the homogeneous agglomerate model ${ }^{6}$. But, it is difficult to understand the relationship between the pore size distribution, the electrolyte fill level, and the polarization, because these models assume a homogeneous pore structure. To understand the relationship, a polarization model with a heterogeneous pore structure model is required.

In this study, the wetting behavior model and the polarization - gas concentration distribution model were developed. The wetting behavior model estimates the reaction surface area as a function of the pore structure and the electrolyte fill level. The polarization - reaction gas concentration model estimates the reaction distribution, the reaction gas concentration distribution, and the polarization as a function of the reaction surface area, the ionic resistance in the electrode, and the operating conditions. These models were analyzed with a half cell measurement ${ }^{7)}$, and then discussed in 
relations to the pore structure, the electrolyte fill level, and the polarization.

\section{THEORY}

\subsection{Wetting behavior model}

The pore structures were assumed to be cylinders of heterogeneous diameters (Fig. 1). When the cumulative pore volume function is $v(r)$ $\left(\mathrm{cm}^{3} / \mathrm{cm}^{3}\right)$, the differential pore volume function is

$$
D(r)=\frac{\partial}{\partial r} V(r)
$$

Here, $r(\mathrm{~cm})$ is the pore radius. The electrode surface area is the summation of the cylinder surface area.

$$
S=\int_{0}^{\infty} \frac{2}{r} \mathrm{D}(r) d r
$$

The contact angle of the cathode and the electrolyte is almost zero. Ther a small amount of the electrolyte will cover the cathode with a thin film. The thickness of the thin film will grow with the increase of the electrolyte, but the film thickness will not exceed the maximum film thickness which is defined by the viscosity and the surface tension of the electrolyte. The wetting process with the electrolyte increase is assumed to include the following steps. i) The cathode is covered by a homogeneous thin electrolyte film. ii) The cathode is covered by a electrolyte film with to maximum thickness. iii) The cathode pores with a smaller size are flooded first.

The total reaction of the cathode is

$$
\mathrm{CO}_{2}+\frac{1}{2} \mathrm{O}_{2}+2 e \rightarrow \mathrm{CO}_{3}^{2-} \text {. }
$$

This reaction is controlled by the mixed diffusion of superoxide ion $\mathrm{O}_{2}^{-}$and $\mathrm{CO}_{2}$ under the operating conditions of the $\mathrm{MCFC}^{8}$ ). The polarization is proportional to the gas / electrolyte interface area for the porous cathode ${ }^{9}$, because the electron transfer rate on the cathode is very fast as high temperature of the MCFC. It is assumed that the

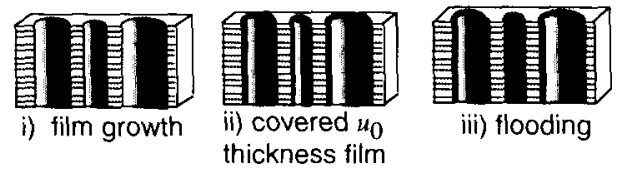

Fig. 1 Pore structure and wetting behavior model. reaction rate is proportional to the gas / electrolyte interface area and the inverse of the electrolyte thickness which is the distance of the diffusion. Then the reaction surface area $\operatorname{sr}\left(\mathrm{cm}^{2} / \mathrm{cm}^{3}\right)$ which is the gas / electrolyte interface area is as follows.

$$
\begin{array}{ll}
S r=\int_{u}^{\infty} \frac{2}{r} D(r)-\frac{2 u}{r^{2}} D(r) d r & \left(u<u_{0}\right) \\
S r=\int_{r,}^{\infty} \frac{2}{r} D(r)-\frac{2 u_{0}}{r^{2}} D(r) d r & \left(r_{f} \geq u_{0}\right)
\end{array}
$$

Here, $u(\mathrm{~cm}), u_{0}(\mathrm{~cm})$, and $r_{f}(\mathrm{~cm})$ are the electrolyte film thickness, the maximum electrolyte film thickness, and the maximum flooded pore radius, respectively. The fill level $f$ which is the electrolyte volume ratio in the cathode pore volume is as follows.

$$
\begin{aligned}
& f=1-\frac{1}{V_{a}} \int_{u i}^{\infty}\left(1-\frac{u}{r}\right)^{2} D(r) d r \quad\left(u<u_{0}\right) \\
& f=1-\frac{1}{V_{r}} \int_{r,}^{\infty}\left(1-\frac{u_{0}}{r}\right)^{2} D(r) d r \quad\left(r_{f} \geq u_{0}\right)
\end{aligned}
$$

Here, $V_{x}$ is porosity which is calculated by the infinite of the cumulative pore volume function $V(\infty)$. Then the reaction surface area is estimated from the pore size distribution and the electrolyte fill level.

\subsection{Polarization Model}

The equivalent circuit of the cathode is shown in Fig. 2. In this figure, $z=0(\mathrm{~cm})$ and $z=$ $z_{0}$ are the current collector side and the electrolyte

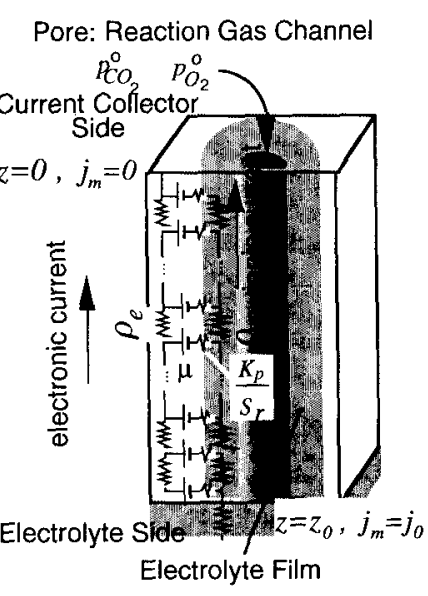

Fig. 2 Polarization model. 
plate side. $\quad \rho_{c}(\Omega \mathrm{cm}), \rho_{m}(\Omega \mathrm{cm})$, and $K_{p}\left(\Omega \mathrm{cm}^{2}\right)$ are the effective electronic resistivity, the effective ionic resistivity, and the interface resistance, respectively. Here, the linear reaction polarization to the load current density is assumed, because the single cell and the half cell polarization is proportional to the load current density ${ }^{10}$.

When Ohm's law is extended to the electrolyte conductivity, the following is obtained

$$
j_{m}=-\frac{1}{\rho_{m}} \frac{\partial \phi_{m}}{\partial z}
$$

Here, $j_{m}\left(\mathrm{~A} / \mathrm{cm}^{2}\right)$ and $\phi_{m}(\mathrm{~V})$ are the ionic current density and the electrolyte potential, respectively.

When the electrode potential, e. m. f., and the reaction current density are $\phi_{e}(\mathrm{~V}), \mu(\mathrm{V})$, and $j_{r}$ $\left(\mathrm{A} / \mathrm{cm}^{2}\right)$, the reaction current density is given as

$$
\phi_{m}-\phi_{i}+\mu=K_{p} j_{r} .
$$

The electronic current density is given by Ohm's law as follows.

$$
j_{e}=-\frac{1}{\rho_{e}} \frac{\partial \phi_{c}}{\partial z}
$$

When the load current density is $j_{0}\left(\mathrm{~A} / \mathrm{cm}^{2}\right)$, the current balance of $j_{r}, j_{e}$, and $j_{m}$ gives the following equations.

$$
\begin{aligned}
& \frac{\partial j_{m}}{\partial z}=-\frac{\partial j_{e}}{\partial z}=-S_{r} j_{r} \\
& j_{m}+j_{e^{\prime}}=j_{0}
\end{aligned}
$$

Equations (9) and (11) give

$$
\frac{\partial j_{m}}{\partial z}=-\frac{S_{r}}{K_{r}}\left(\phi_{m}-\phi_{i}+\mu\right)
$$

Equations (8), (10), (12), and (13) give the following differential equation.

$$
\frac{\partial^{2} j_{m}}{\partial z^{2}}-\frac{S_{r}}{K_{p}}\left\{\left(\rho_{m}+\rho_{c}\right) j_{m}+\frac{\partial \mu}{\partial z}-\rho_{\imath} j_{0}\right\}=0
$$

Because of the current balance, the ionic current density which is the boundary condition of eq. (14) is

$$
\left.j_{m}\right|_{z=0}=0,\left.j_{m}\right|_{z=z_{0}}=j_{0} .
$$

$\frac{\partial \mu}{\partial z}$ is the differential of the Nernst equation,

$$
\frac{\partial \mu}{\partial z}=-\frac{R T}{2 F} \frac{\partial}{\partial z} \ln \left(p_{C O_{2}} p_{O_{2}} \frac{1}{2}\right)
$$

The dependence of the apparent reaction resistance on the atmosphere is small ${ }^{10}$ ). It is assumed that the dependence of the interface resistance on the atmosphere is negligible and is proportional to film thickness.

$$
K_{p}=u K_{p}^{0} \quad\left(0<u \leq u_{0}\right)
$$

Here, $K_{p}^{0}(\Omega \mathrm{cm})$ is the standard interface resistance.

The apparent reaction resistance $R_{p}\left(\Omega \cdot \mathrm{cm}^{2}\right)$ can be calculated from the potential difference of the equivalent circuit.

$$
R p=\frac{\left|\frac{K_{r}}{S_{r}} \frac{\partial j_{m}}{\partial z}\right|_{i=0}+\int_{0}^{i 0} j_{m} \rho_{m} d z \mid}{j_{0}}
$$

The ionic current and the electronic current path are tortuously. The effective ionic resistivity is

$$
\rho_{m}=\frac{\rho_{m}^{0}}{W^{t}} \text {. }
$$

Here, $\rho_{m}^{0}, W$, and $x$ are the species ionic resistivity of the electrolyte, the electrolyte volume ratio per unit volume, and the tortuosity. The electronic resistivity is estimated by the same treatment.

\section{3 Reaction Gas Concentration Distribution model}

The partial pressure of reaction gas is defined by the balance between concentration diffusion, consumption by the reaction, and the flux of the reaction consumption which is flowed by the volume change with the reaction at the electrolyte plate side (Fig. 3).

When the effective diffusion coefficient of the carbon dioxide is $D_{\mathrm{CO}_{2}}\left(\mathrm{~cm}^{2} / \mathrm{s}\right)$, the partial pressure change of the carbon dioxide by the diffusion is given by

$$
\frac{\partial p_{C O_{2}}}{\partial t}=D_{C O_{2}} \frac{\partial^{2} p_{C O_{2}}}{\partial z^{2}} .
$$

Here, $p_{C O}(\mathrm{~atm})$ is the partial pressure of the carbon dioxide.

The partial pressure change due to consumption of species is as follows. The characteristic equation for the unit volume of the electrode is

$$
p_{\mathrm{CO}_{2}}\left(V_{\infty}-W\right)=n R T .
$$

Here, $n(\mathrm{~mol}), R(\mathrm{~kJ} / \mathrm{mol} \mathrm{K})$, and $T(\mathrm{~K})$ are the molar 


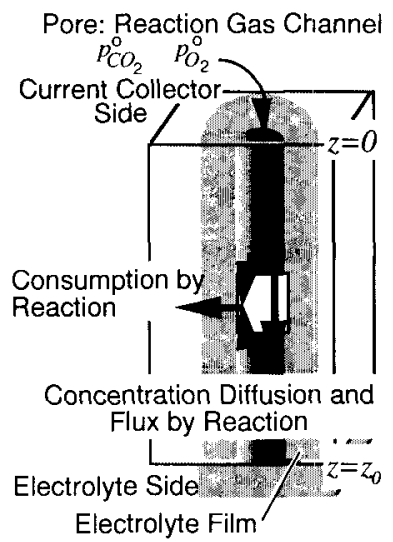

Fig. 3 Concentration distribution model.

quantity, the gas constant, and the temperature, respectively.

The time differential of eq. (21) is

$$
\begin{aligned}
\left(V_{\infty}-W\right) \frac{\partial p_{\mathrm{CO}_{2}}}{\partial t} & =R T \frac{\partial t}{\partial t} \\
& =\frac{R T}{2 F} S_{r} j_{r} \\
& =-\frac{R T}{2 F} \frac{\partial j_{m}}{\partial z} \\
\therefore \frac{\partial p_{\mathrm{CO}_{2}}}{\partial t} & =-\frac{R T}{2 F\left(V_{\infty}-W\right)} \frac{\partial j_{m}}{\partial z} .
\end{aligned}
$$

Here, $F(\mathrm{C} / \mathrm{mol})$ is the Faraday constant.

The carbon dioxide flux is given by the reaction volume of the electrolyte plate side. When the carbon dioxide flux is $J_{\mathrm{CO}_{2}}\left(\mathrm{~mol} / \mathrm{s} \mathrm{cm}^{2}\right)$, the flux is

$$
J_{\mathrm{CO}_{2}}=\frac{3}{2} \frac{1}{2 \mathrm{~F}\left(V_{\infty}-W\right)}\left(j_{0}-j_{m}\right) p_{\mathrm{CO}_{2}}
$$

The partial pressure change by the flux is given by the distance differential of the flux.

$$
\begin{aligned}
\frac{\partial p_{\mathrm{CO}_{2}}}{\partial t} & =-R T \frac{\partial J_{\mathrm{CO} 2}}{\partial z} \\
& =-\frac{3}{2} \frac{R T}{2 F\left(V_{\infty}-W\right)}\left\{\left(j_{0}-j_{m}\right) \frac{\partial p_{\mathrm{CO}_{2}}}{\partial z}-p_{\mathrm{CO}_{2}} \frac{\partial j_{m}}{\partial z}\right\}
\end{aligned}
$$

The partial pressure change is the summation of eq. (20), (22), and (24).

$$
\begin{aligned}
\frac{\partial p_{C O_{2}}}{\partial t}= & D_{C O_{2}} \frac{\partial^{2} P_{C O_{2}}}{\partial z^{2}}-\frac{R T}{2 F\left(V_{\infty}-W\right)} \frac{\partial j_{m}}{\partial z} \\
& -\frac{3}{2} \frac{R T}{2 F\left(V_{\infty}-W\right)}\left\{\left(\dot{0}-j_{m}\right) \frac{\partial p_{C O_{2}}}{\partial z}-p_{C O_{2}} \frac{\partial j j_{m}}{\partial z}\right\}
\end{aligned}
$$

In steady state, the carbon dioxide partial pressure is not change, then eq. (25) is

$$
\begin{aligned}
D_{\mathrm{CO}_{2}} & \frac{\partial^{2} p_{\mathrm{CO}_{2}}}{\partial z^{2}}-\frac{R T}{2 F\left(V_{\infty}-W\right)} \frac{\partial j_{m}}{\partial z} \\
- & \frac{3}{2} \frac{R T}{2 F\left(V_{\infty}-W\right)}\left\{\left(j_{0}-i_{m}\right) \frac{\partial p_{\mathrm{CO}_{2}}}{\partial z}-p_{\mathrm{CO}_{2}} \frac{\partial j_{m}}{\partial z}\right\}=0
\end{aligned}
$$

The boundary conditions are follows; the partial pressure at the current collector side is the supplied partial pressure, and there is no partial pressure gradient at the current collector side.

$$
\left.p_{\mathrm{CO}_{2}}\right|_{z=0}=p_{\mathrm{CO}_{2}}^{0},\left.\frac{\partial p_{\mathrm{CO}_{2}}}{\partial z}\right|_{z=0}=0
$$

The partial pressure change of the oxygen is determined by the same way. When the effective diffusion coefficient of the oxygen is $D_{O_{2}}\left(\mathrm{~cm}^{2} / \mathrm{s}\right)$

$$
\begin{aligned}
& D_{\mathrm{O}_{2}} \frac{\partial^{2} p_{\mathrm{O}_{2}}}{\partial z^{2}}-\frac{R T}{4 F\left(V_{\infty}-W\right)} \frac{\partial j_{m}}{\partial z} \\
& \quad-\frac{3}{2} \frac{R T}{2 F\left(V_{\infty}-W\right)}\left\{\left(j_{0}-j_{m}\right) \frac{\partial p_{\mathrm{O}_{2}}}{\partial z}-p_{\mathrm{O}_{2}} \frac{\partial j_{m}}{\partial z}\right\}=0
\end{aligned}
$$

$$
\left.p_{\mathrm{O}_{2}}\right|_{z=0}=p_{\mathrm{O}_{2}}^{0},\left.\frac{\partial p_{\mathrm{O}_{2}}}{\partial z}\right|_{z=0}=0
$$

The effective diffusion coefficient is determined by

$$
D_{i}=D_{i}^{0}\left(1-V_{\infty}-W\right)^{\prime \prime}
$$

Here, $D_{i}\left(\mathrm{~cm}^{2} / \mathrm{s}\right), D_{i}^{0}\left(\mathrm{~cm}^{2} / \mathrm{s}\right)$, and $y$ are theeffective diffusion coefficient of species $i$, the molecular diffusion coefficient of species $i$ and the gas diffusion tortuosity.

\section{METHOD OF ANALYSIS}

The electrode pore size distributions for the analysis are shown in Fig. 4. Polarization of electrode $C$ was measured by the half cell measurement using a lithiated nickel oxide electrode with lithium / potassium $=62 / 38 \mathrm{~mol} \%$ eutectic carbonate electrolyte at $923 \mathrm{~K}$ under $70 \%$ Air - $30 \% \mathrm{CO}_{2}{ }^{7}$ ). Electrodes $\mathrm{A}, \mathrm{B}$, and $\mathrm{D}$ have different median pore diameters with the same pore size distribution profile. The properties of the electrodes are shown in Table 1 . The porosity 


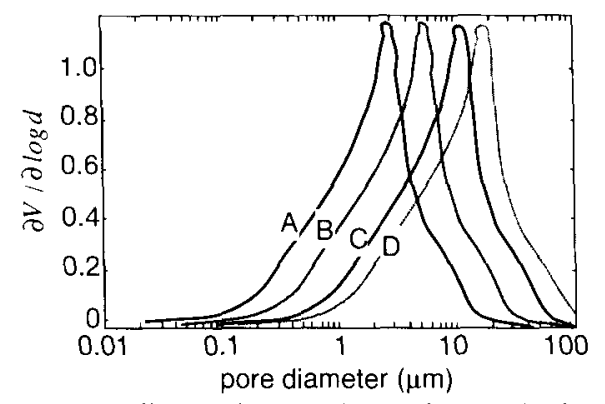

Fig. 4 Differential pore volume of the cathodes.

Table 1 The cathode properties.

\begin{tabular}{ccccc} 
& A & B & C & D \\
\hline $\begin{array}{c}\text { Medium Pore } \\
\text { Diameter( } \mu \mathrm{m})\end{array}$ & 1.8 & 3.7 & 7.3 & 11.0 \\
$\begin{array}{c}\text { Surface Area } \\
\left(\mathrm{m}^{2} / \mathrm{cm}^{3}\right)\end{array}$ & 2.6 & 1.5 & 0.8 & 0.6 \\
\hline
\end{tabular}

Table 2 The resistivities and the diffusion coefficients at $650^{\circ} \mathrm{C}$

\begin{tabular}{lll}
\hline$\rho_{m}^{0} 0.72 \Omega \mathrm{cm}$ & $D_{\mathrm{CO}_{2}}^{0}$ & $1.35 \mathrm{~cm}^{2} / \mathrm{s}$ \\
$\rho_{c}^{0} 3.03 \times 10^{-2} \Omega \mathrm{cm}$ & $D_{O_{2}}^{0}$ & $1.38 \mathrm{~cm}^{2} / \mathrm{s}$ \\
\hline
\end{tabular}

was $50 \%$, and the thickness was $0.06 \mathrm{~cm}$ for all electrodes. The operating conditions for the calculation were equal to those for the half cell measurement ${ }^{7}$ ). The species resistivities and the molecular diffusion coefficients are shown in Table $2^{10-12)}$. The maximum thickness of the electrolyte film was assumed to be $1.0 \mu \mathrm{m}$ by the cross section of the operated electrode ${ }^{9}$ ). The standard interface resistance and tortuosities is fitting parameters. They were determined to fit the calculation with the half cell measurements ${ }^{7)}$.

The solution of the model equations was carried out on Math matica ${ }^{\mathrm{B}}$ version 3.01 which is a system for doing mathematics by computer.

The relationship between the reaction surface area, the fill level, and the pore sizedistribution was determined by equations (4), (5), (6), and (7) using the integration function of Mathmatica ${ }^{\sqrt{ }}$.

The ionic current distribution and the partial pressure distribution of the reactant gases were determined by the simultaneous differential equations (14), (15), (26), (27), (28), and (29). The solution of the differential equations was carried out by the finite difference method.

\section{RESULTS AND DISCUSSION}

\subsection{The reaction surface area}

The reaction surface area as a function of the fill level for each electrode is show in Fig. 5. The electrode with smaller median pore diameter which has a larger specific surface area has a larger reaction surface area. The reaction surface area decreases with increased fill level. The fill level in the MCFC is defined by the balance of the capillary force between the cathode, anode, and electrolyte matrix. To optimize the cathode pore structure design, the anode pore structure design must be optimized. If the anode pore structure is not optimized, it is possible that a electrode with larger median pore diameter has a larger reaction surface area than the smaller median pore diameter electrode when they have the same flooded pore radius and the flooded pore radius is too large for

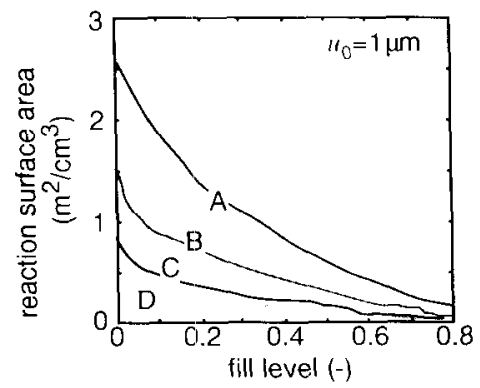

Fig. 5 Reaction surface area as a function of fill level.

the smaller median pore diameter electrode.

4.2 The relationship between the reaction resistance and the electrolyte fill level

The calculation results of the relationship between the apparent reaction resistance and the fill level for the electrode $C$ is shown in Fig. 6 with the half cell measurements. The standard interface resistance, the resistance totorisity, and the gas diffusion totorisity are $1.0 \times 10^{\circ}(\Omega \mathrm{cm}), 1.5$, and 1.5 , respectively. The calculation is fitted to the measurement by these parameters.

In the region of the fill level shortage, the apparent reaction resistance decreases with the increased fill level. In the region of the suitable fill level, it hardly changes. In the region of the excess fill level, it increases with increased fill 


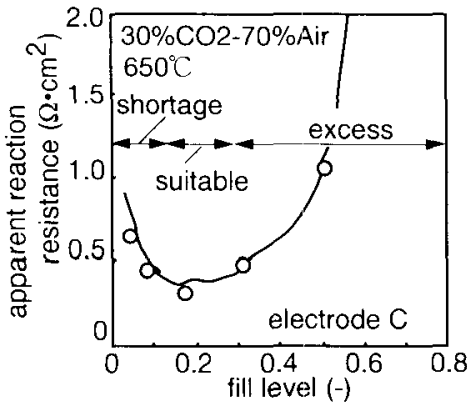

Fig. 6 The relationship between the reaction resistance and the fill level with Li/K carbonate .

Line: calculated; Plots: measured

level.

Since the observed and the calculated results are in good agreement, the reaction behavior in the electrode and the different pore structure electrode can be analyzed by these parameters.

Figure 7 shows the reaction current profiles in the electrodes. Fill level $=0.09$ is an example of the fill level shortage. In the region of the fill level shortage, the reaction is concentrated at the electrolyte side because of the high ionic resistance. Then the apparent reaction resistance decreases with fill level increase, which leads to the ionic resistance decrease.

Fill level $=0.21$ is an example of the suitable fill level. In the region of the suitable fill level, reaction is not concentrated and not uniform. The profile is defined by the balance of the ionic resistivity and the reaction resistance. This profile has a the relaxation effect for the apparent reaction resistance change by the change of the electrolyte fill level with reaction distribution change. Then the apparent reaction resistance hardly changes.

Fill level $=0.41$ is an example of the excess fill level. In the region of the excess fill level, reaction is uniform because of the low ionic resistance and the high interface resistance. Then the apparent reaction resistance increases with fill level increase, which leads to the interface resistance increase

The potential profiles in the electrode with various electrolyte fill level are shown in Fig. 8. The potential profiles are the parameter of the gas concentration distribution. The potential difference increases with the increased electrolyte fill level due to the uniform reaction current profiles and the smaller diffusion path for the higher electrolyte fill level.

The value of the potential difference is very small for the electrolyte shortage and suitable region. The polarization effect of the potential difference is negligible except for the region of the excess fill level.

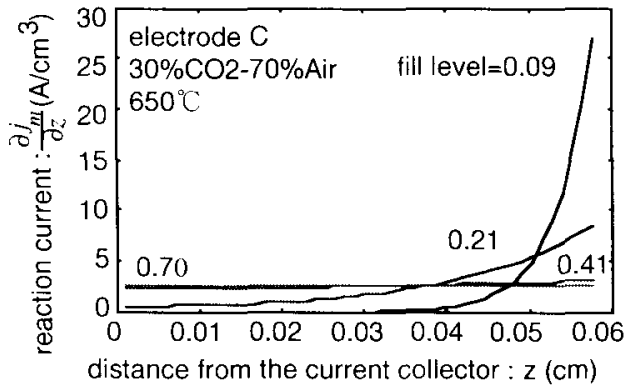

Fig. 7 Reaction current profiles.

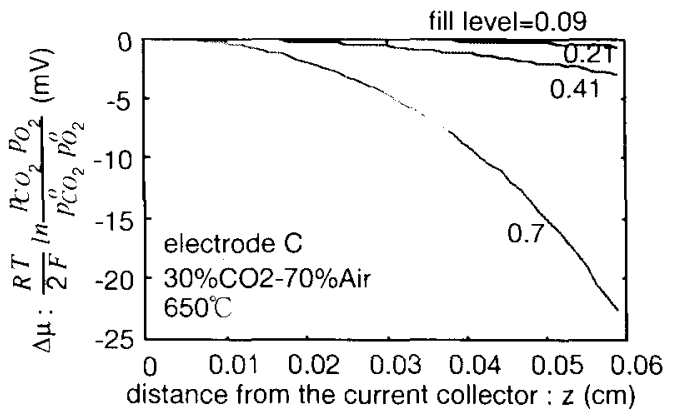

Fig. 8 Potential difference profiles.

4.3 The relationship between the reaction resistance and the median pore diameter

The fitting parameters are determined for electrode C. Figure 9 shows calculated curves for electrodes with different median pore diameter. The smaller median pore diameter electrode has good performance. The electrode with the smaller median pore diameter has a smaller apparent reaction resistance because of the larger reaction surface area. In the region of the fill level shortage, the apparent reaction resistances are almost the same values for each electrode. The electrode with smaller median pore diameter has a wider suitable fill level region.

Figure 10 shows the reaction current profiles for 


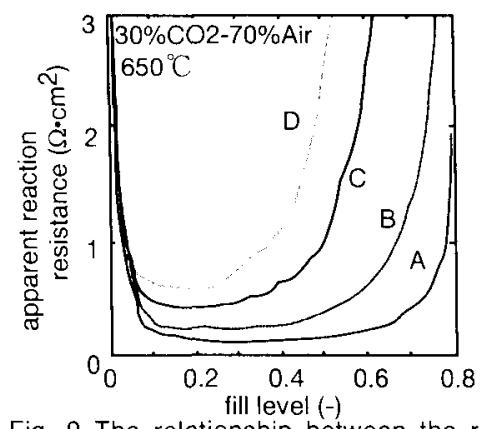

Fig. 9 The relationship between the reaction resistance and the fill level with $\mathrm{Li} / \mathrm{K}$ carbonate .

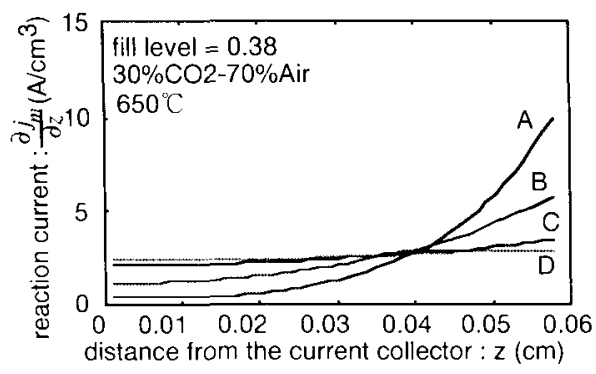

Fig. 10 Reaction current profiles.

each electrode at the electrolyte fill level $=0.38$. At this point, electrodes $A$ and $B$ are in the region of the suitable fill level, and electrodes $C$ and D are in the region of the fill level shortage.

Electrodes $A$ and $B$ do not have a concentrated or uniform reaction current distribution. Their profiles have the relaxation effect for the apparent reaction resistance change due to the electrolyte fill level change. Then they are in the region of the suitable fill level. Electrodes $C$ and $D$ have uniform reaction current distributions, because they are in the region of the excess fill level.

The reason why the smaller median pore diameter electrode does not have a uniform reaction current profile is that it has a smaller interface resistance, because of the larger reaction surface area at higher electrolyte fill level.

\section{CONCLUSION}

The polarization model for the molten carbonate fuel cell cathode has been investigated to understand the relationship between the pore size distribution, the electrolyte fill level, and the polarization.
The smaller median pore diameter electrode had smaller reaction resistance and wider suitable quantity electrolyte region, because of the larger reaction surface area. The reaction current profile in the suitable quantity electrolyte region was determined by the balance of the ionic resistivity and the reaction resistance.

This work was conducted under contract from the MCFC Research Association. The MCFC Research Association was commissioned to do the work by NEDO (New Energy and Industrial Technology Development Organization) as a part of the New Sunshine Program of MITI (Ministry of International Trade and Industry). We appreciate the advice and support of MCFC Research Association, NEDO, and MITI.

\section{REFERENCES}

1) S. Mitsushima, K. Yamaga, H. Okada, and T. Kamo, Denki Kagnku, 65, 395(1997).

2) Y. Fujita, H. Urushibata, and A. Sasaki, Denki Kagnku, 65, 565(1997).

3) K. Tanimoto, M. Yanagida, T. Kojima, Y. Tamiya, and Y. Miyazaki, Proceeding of the 2nd International Fuel Cell Conference, p.207 (1996).

4) Y. Mugikura, T. Abe, Y. Izaki, S. Yoshioka, and H. Urushibata, Denki Kagnku, 62, 624 (1994).

5) G. Wilemski, J. Electrochem. Soc., 130, 117(1983).

6) C. Y. Yuh and J. R. Selman, I. Electrochem. Soc., 131, 2062(1984).

7) Y. Iwase, S. Kuroe, and S. Mitsushima, Denki Kagnku, 62, 152(1994).

8) T. Nishina, I. Uchida, and J. R. Selman, J. Electrocient. Soc, 141, 1191 (1994).

9) S. Kuroe, M. Takeuchi, S. Nishimura, and K. Ohtsuka, Denki Kagaku, 58, 928 (1990).

10) C. Y. Yuh and J. R. Selman, I. Electrochem. Soc., 138, 3642 (1991).

11) C. E. Baumgartner, R. H. Arendt, C. D. Iacovangelo, and B. R. Karas, J. Electroclem. Soc., 131, 2217 (1984).

12) P. L. Spedding, /. Electrochem. Soc., 120, 1049(1973).

13) S. Fujita, Kngaku Kougaku, 28, 251 (1964). 